\title{
Cannabinoids and Glucocorticoids in the Basolateral Amygdala Modulate Hippocampal-Accumbens Plasticity After Stress
}

\author{
Amir Segev' and Irit Akirav*,I \\ 'Department of Psychology, University of Haifa, Haifa, Israel
}

\begin{abstract}
Acute stress results in release of glucocorticoids, which are potent modulators of learning and plasticity. This process is presumably mediated by the basolateral amygdala (BLA) where cannabinoids $C B I$ receptors have a key role in regulating the hypothalamic-pituitaryadrenal (HPA) axis. Growing attention has been focused on nucleus accumbens (NAc) plasticity, which regulates mood and motivation. The NAc integrates affective and context-dependent input from the BLA and ventral subiculum (vSub), respectively. As our previous data suggest that the CBI/2 receptor agonist WIN55,2 I2-2 (WIN) and glucocorticoid receptor (GR) antagonist RU-38486 (RU) can prevent the effects of stress on emotional memory, we examined whether intra-BLA WIN and RU can reverse the effects of acute stress on NAc plasticity. Bilateral, ipsilateral, and contralateral BLA administration of RU or WIN reversed the stress-induced impairment in vSub-NAc long-term potentiation (LTP) and the decrease in CAMP response element-binding protein (CREB) activity in the NAc. BLA CBI receptors were found to mediate the preventing effects of WIN on plasticity, but not the preventing effects of RU, after stress. Inactivating the ipsilateral BLA, but not the contralateral BLA, impaired LTP. The possible mechanisms underlying the effects of BLA on NAc plasticity are discussed; the data suggest that BLA-induced changes in the NAc may be mediated through neural pathways in the brain's stress circuit rather than peripheral pathways. The results suggest that glucocorticoid and cannabinoid systems in the BLA can restore normal function of the NAc and hence may have a central role in the treatment of a variety of stress-related disorders.

Neuropsychopharmacology (2016) 4I, I066-1079; doi:I0.1038/npp.20I5.238; published online I6 September 2015
\end{abstract}

\section{INTRODUCTION}

Stress influences the brain by modifications in neuronal plasticity. The brain's stress circuit (ie, amygdala-hippocampal-cortico-striatal) has been suggested as a key circuit responsible for processing and storing emotion-related memories and for coordinating stress-related behaviors.

The nucleus accumbens (NAc) integrates limbic and cortical inputs arising from monosynaptic glutamatergic projections that originate in the ventral subiculum of the hippocampus (vSub) regarding context dependency, the basolateral amygdala (BLA) regarding affective responses, and the medial prefrontal cortex (mPFC) regarding behavioral flexibility. Monosynaptic BLA and vSub projections converge on the distal dendrites and spines of NAc neurons, and it has been suggested that the BLA modulates plasticity in the vSub-NAc pathway (Gill and Grace, 2011). Moreover, the NAc may be a site of convergence of BLA and hippocampal influences in modulating memory consolidation, as NAc lesions blocked the memory-enhancing effect of intra-BLA or intrahippocampal glucocorticoid receptor (GR) agonist on inhibitory avoidance consolidation (Roozendaal et al, 2001; Setlow et al, 2000).

*Correspondence: Professor I Akirav, Department of Psychology, University of Haifa, Mt Carmel, Haifa 31905, Israel, Tel: +972 4 8288268, Fax: +972 48263 I57, E-mail: irit.akirav@gmail.com

Received 2 March 2015; revised 3 August 2015; accepted 4 August 2015; accepted article preview online 20 August 2015
The endocannabinoid (eCB) system is important for mood regulation, anxiety, and fear extinction (Abush and Akirav, 2013; Ganon-Elazar and Akirav, 2009, 2012, 2013; Marsicano et al, 2002; Moreira and Wotjak, 2010; Patel and Hillard, 2006; Viveros et al, 2005). We found that the CB1/2 receptor agonist WIN55,212-2 (WIN) prevented the effects of stress on learning and plasticity, as well as the behavioral and neuroendocrine outcomes of stress (Abush and Akirav, 2013; Ganon-Elazar and Akirav, 2009, 2012, 2013). Specifically, systemic administration of WIN or the GR antagonist RU-38486 (RU) prevented the impairment in extinction and NAc plasticity induced by exposure to chronic mild stress (Segev et al, 2014), and intra-BLA WIN or RU administered before stress exposure prevented the stress-induced enhancement of memory consolidation for reduction in reward magnitude (Ramot and Akirav, 2012).

Studies indicate a bidirectional, functional relationship between glucocorticoids and eCBs (for a review, see: Akirav, 2013). ECBs have a key role in regulating the hypothalamicpituitary-adrenal (HPA) axis under basal and stressful conditions (Ganon-Elazar and Akirav, 2012, 2013; Hill et al, 2009; Patel et al, 2004). Stress and glucocorticoids can trigger eCB synthesis and $\mathrm{CB} 1$ receptors signaling to constrain HPA axis activity under acute conditions (Hill et al, 2011; Rademacher et al, 2008; Steiner and Wotjak, 2008). Blocking BLA GRs prevented the effects of stress on a variety of memory tasks (Maroun and Akirav, 2007; Roozendaal et al, 2002; Segev et al, 2012, 2014). 
Changes in the activity of CREB were related to synaptic plasticity, learning and memory, and antidepressants effects (Bourtchuladze et al, 1994; Nestler et al, 2002). Stress increased NAc cAMP response element (CRE)-dependent transcription (Barrot et al, 2002; Pliakas et al, 2001). However, it has been suggested that anxiety associated with novelty correlates with a decrease in NAc CRE-mediated transcription, and increasing NAc CRE activity following stress can rescue an anxiety-like phenotype (Barrot et al, 2005).

In this study, we examined whether stress could alter NAc long-term potentiation (LTP) and phospho (p)CREB expression, a well-defined mechanism for neural plasticity. We also examined whether BLA cannabinoid activation or GR deactivation after stress exposure would reverse the stress-induced alterations in NAc plasticity and pCREB. Given the role of the NAc in processing the rewarding and emotional salience of stimuli, stress-induced alterations in the modulation of BLA and vSub responses in the NAc could impact the normal filtering of NAc responses and consequently behavior.

\section{MATERIALS AND METHODS}

\section{Subjects}

Male Sprague-Dawley rats (Harlen, 300-350 g) were group housed at $22 \pm 2{ }^{\circ} \mathrm{C}$ under 12-h light/dark cycles (lights turned on at 0700 hours). Rats had access to water and laboratory rodent chow ad libitum. The experiments were approved by the University of Haifa Ethics and Animal Care Committee, and adequate measures were taken to minimize pain or discomfort (permit number: 116).

\section{Drugs}

RU-38486 (RU; 10 ng/0.5 $\mu \mathrm{l} /$ side) (Sigma, IL) was dissolved in $2 \%$ ethanol and $98 \%$ saline. WIN $(5 \mu \mathrm{g} / 0.5 \mu \mathrm{l} /$ side $)$ and AM251 (0.3 ng/0.5 $\mu \mathrm{l} /$ side) (Cayman Chemical) were dissolved in $1 \%$ dimethyl sulfoxide (DMSO), $1 \%$ Tween-80, and $98 \%$ saline. Controls were given the vehicle (Veh) only. Muscimol $(0.03 \mathrm{nmol})$ (MP Bio, Solon, $\mathrm{OH})$ and baclofen $(0.3 \mathrm{nmol})$ (Santa Cruz Biotechnology, CA) were dissolved in saline.

Drug concentrations were based on previous results (Ganon-Elazar and Akirav, 2009, 2012, 2013; Maroun and Akirav, 2007; Ramot and Akirav, 2012).

\section{Elevated Platform Stress}

Animals were placed on an elevated platform (EP; $12 \times 12 \mathrm{~cm}$ ) for $30 \mathrm{~min}$ in a brightly lit room. The rats exhibited behavioral 'freezing', that is, immobility for up to $10 \mathrm{~min}$, defecation, and urination (Ganon-Elazar and Akirav, 2009; Maroun and Akirav, 2007; Segev et al, 2012).

\section{Microinjection}

Rats were anesthetized (40\% urethane, 5\% chloral hydrate in saline, $0.5 \mathrm{ml} / 100 \mathrm{~g}$, i.p.), placed in a Stoelting stereotaxic frame, and microinjected into the BLA (coordinates: $2.7 \mathrm{~mm}$ posterior, $5 \mathrm{~mm}$ lateral, and $6.2 \mathrm{~mm}$ ventral to bregma).

For microinjection, a stainless steel guide cannula (23 gauge, thin wall) was lowered to the BLA and a 28-gauge injection cannula, extending $1.0 \mathrm{~mm}$ from the tip of the guide cannula, was inserted through it. The injection cannula was connected via PE20 tubing to a Hamilton micro syringe driven by a micro infusion pump (PHD 100, Harvard Apparatus). Microinjection was performed in a $0.5-\mu 1$ volume delivered over $2 \mathrm{~min}$. The injection cannula was left in position for an additional 2 min before withdrawal in order to minimize dragging of the injected liquid along the injection tract.

\section{Electrophysiology}

Rats were prepared for electrophysiological recording as described previously (Korem and Akirav, 2014). A bipolar concentric stimulating electrode was implanted in the vSub $(6.5 \mathrm{~mm}$ posterior, $5 \mathrm{~mm}$ lateral, and $6 \mathrm{~mm}$ ventral to bregma). A recording microelectrode (glass, tip diameter 2-5 $\mu \mathrm{m}$, filled with $2 \mathrm{M} \mathrm{NaCl}$, resistance 1-4 $\mathrm{M} \Omega$ ) was inserted in the ipsilateral NAc shell $(1.6 \mathrm{~mm}$ anterior, $1 \mathrm{~mm}$ lateral, $5 \mathrm{~mm}$ ventral to bregma). Evoked field potentials (EFPs) were amplified $(\times 1000)$ by an A-M Systems amplifier, digitized at $10 \mathrm{kHz}$, and stored on disk for off-line analysis using the Cambridge Electronic Design (CED, Cambridge, UK) $1401+$ and Spike2 software.

After positioning of the electrodes, the animal was left undisturbed for $30 \mathrm{~min}$ to allow the tissue to settle. Baseline stimuli to the vSub (monophasic pulses, 100-ms duration, intensity adjusted to yield a field potential of $40 \%$ of the maximal pretetanus value in NAc) were delivered at $0.1 \mathrm{~Hz}$ for $30 \mathrm{~min}$, after which LTP was induced by a theta-like highfrequency stimulation (HFS) to the vSub (HFS: three sets of 10 trains, each train consisting of 10 pulses at $200 \mathrm{~Hz}$; intertrain interval: $200 \mathrm{~ms}$; interset interval: $1 \mathrm{~min}$ ). Responses to baseline stimulation every $10 \mathrm{~s}$ were then collected for $1 \mathrm{~h}$. LTP was measured as an increase in the amplitude and slope of the EFPs. Potentiation was measured as a percentage change from the average of the $30 \mathrm{~min}$ baseline before HFS.

\section{Biochemical Methods}

Western blots. Rats were killed and their brains were frozen at $-80^{\circ} \mathrm{C}$ for western blotting analysis. Brain tissues was cryosectioned and the NAc shell, BLA, and the medial amygdala (MeA) were collected and homogenized in lysis buffer (10 mM Hepes, $2 \mathrm{mM}$ EDTA, $2 \mathrm{mM}$ EGTA, $0.5 \mathrm{mM}$ DTT, $10 \mu \mathrm{l} / \mathrm{ml}$ leupeptin, $10 \mu \mathrm{l} / \mathrm{ml}$ aprotinin). Protein levels were determined by Bicinchoninic Acid Protein Assay Kit (Thermo Scientific, Waltham, MA) according to the manufacturer's protocol. The samples were then diluted in SDS sample buffer, boiled $\left(100^{\circ} \mathrm{C}\right)$ for $5 \mathrm{~min}$, and stored at $-80^{\circ} \mathrm{C}$. Aliquots were subjected to SDS-PAGE $(10 \%$ polyacrylamide) and immunoblot analysis. Blots were blocked overnight with BSA and were then incubated overnight with CREB or pCREB (Ser133) primary antibody at $4{ }^{\circ} \mathrm{C}(1: 1000$, Cell Signaling), followed by washing and $1 \mathrm{~h}$ incubation with an HRP-linked secondary antibody at room temperature (goat anti-rabbit IgG; Jackson ImmunoResearch Laboratories, $1: 10000$ ). Blots were visualized by enhanced chemiluminescence with ECL (Biological Industries) and quantified with an XRS charge-coupled device camera (Bio-Rad, Hercules, CA) and Quantity One software. 
RNA extraction, cDNA preparation, and gene expression quantification. Thirty minutes after exposure to EP stress, rats were decapitated and their brains were frozen at $-80^{\circ} \mathrm{C}$ for cFOS RNA analysis. The BLA and the NAc were punched.

RNA extraction and cDNA preparation were performed using standard methodology, as previously described (Zaidan et al, 2013). mRNA gene expression levels of C-fos were determined by real-time quantitative PCR (qRT-PCR), using SYBR-Green fluorometric-based detection as previously described (Zaidan et al, 2013). Primers were designed using Primer Express (Applied Biosystems, Foster City, CA) and generated by Integrated DNA Technologies (Coralville, IA). The $\Delta \Delta \mathrm{Ct}$ method was used to calculate differences in mRNA expression: intensity values for each gene were normalized to Hprt $(\Delta \mathrm{Ct})$. Fold change was calculated as $2^{-\Delta \Delta C t}$ relative to a control group in each region.

Ratios of C-fos were determined using the comparative $\mathrm{Ct}$ equation with minor modifications, as described previously (Gordon et al, 2003). Calculation of an expression ratio using data from two genes in any single sample obviates the need for a calibrator sample and a reference gene for standardization. Thus the $\Delta \Delta \mathrm{CT}$ value in the comparative CT equation was expressed as (CT(gene 1) - CT(gene 2)). All primers and reagents were purchased from Biosearch technology. The forward and reverse primers used were the followings: c-Fos forward 5'-GATACGCTCCAAGCGGAGAC-3' and reverse 5'-GGCTGCCAAAATAAACTCCAGTT-3'.

\section{Statistics}

The results are expressed as means \pm SEM. For statistical analysis, two-way ANOVA, mixed design three-way and two-way ANOVA, and independent-samples $t$-test were used as indicated. All post-hoc comparisons were made using the least significant difference multiple-comparison test.

\section{RESULTS}

The Effects of Bilateral Intra-BLA RU-38486, WIN55,212-2, or AM251 on vSub-NAc LTP After Stress

All electrophysiological measures were analyzed using mixed design three-way ANOVA post-HFS (Drug $\times$ Stress $\times$ Time
$(2 \times 2 \times 12))$ or pre-HFS $(2 \times 2 \times 5)$. In all electrophysiological experiments analysis pre-HFS did not reveal any significant effects for Drug, Stress, Time, or any of the interactions between them, suggesting a similar baseline between the groups before HFS was applied.

We examined whether intra-BLA RU or WIN would reverse the effects of EP stress on plasticity (Figure 1a). Analysis on amplitude (Figure 1b) and slope (Figure 1c) revealed a significant Drug $\times$ Stress interaction (amplitude: $\mathrm{F}_{(1,22)}=25.195, P<0.001$; slope: $\left.\mathrm{F}_{(1,22)}=9.73, P<0.01\right)$ and a significant Time $\times$ Stress interaction (slope: $\mathrm{F}_{(1,22)}=14.36$, $P=0.001)$. There were no significant effects for Drug, Stress, Time, Time $\times$ Drug interaction, or Time $\times$ Drug $\times$ Stress interaction.

Post-hoc comparison revealed significant differences between the Stress-Vehicle group and the No StressVehicle (amplitude: $P<0.001$; slope: $P<0.05$ ) and the Stress-RU (amplitude: $P=0.012$; slope: $P<0.01$ ) groups and between the No Stress-RU group and the No StressVehicle (amplitude: $P=0.001$ ) and the Stress-RU (amplitude: $P<0.05)$ groups. Hence, stress impaired LTP compared with no stress and bilateral intra-BLA RU reversed this stressinduced impairment. Bilateral intra-BLA RU with no stress also impaired LTP.

To examine the effects of stress and RU on baseline synaptic activity, input-output measurements were taken. Mixed design three-way ANOVA $($ Stress $\times$ Drug $\times$ Intensity $(2 \times 2 \times 5))$ revealed a significant effect for stimulation intensity on amplitude (Figure 1d; $\mathrm{F}_{(1,22)}=308.46, P<0.001$ ). Yet, stimulation of the vSub input into the NAc with different stimulus intensities did not result in any other significant effects on basal EFPs, suggesting no effect on baseline synaptic activity.

Next we examined whether bilateral intra-BLA WIN would reverse the effects of EP stress on plasticity. Analysis on amplitude (Figure 1e) and slope (Figure 1f) post-HFS revealed significant effects for Stress (amplitude: $\left.\mathrm{F}_{(1,22)}=6.17, \quad P<0.05\right)$, Drug (amplitude: $\mathrm{F}_{(1,22)}=5.28$, $P<0.05$; slope: $\left.\mathrm{F}_{(1,22)}=5.14, P<0.05\right)$, Drug $\times$ Stress interaction (amplitude: $\mathrm{F}_{(1,22)}=15.49, P=0.001$ ), and Time $\times$ Stress interaction (slope: $\mathrm{F}_{(1,22)}=8.39, P<0.01$ ).

Post-hoc comparison revealed a significant difference between the Stress-Vehicle group and the No StressVehicle (amplitude: $P<0.001$; slope: $P=0.05$ ) and the

\footnotetext{
Figure I The effects of bilateral intra-BLA GR deactivation and CBI/2 receptor activation on synaptic plasticity in the vSub-NAc pathway after stress. (a) Rats were exposed to the EP stress for 30 min or not, followed by anesthesia (Anes.) and bilateral intra-BLA microinjections (BLA Microinject.) of vehicle, $\mathrm{RU}, \mathrm{WIN}$, or AM25I. Drug microinjection was performed $15 \mathrm{~min}$ after the stressor ended. Rats were then taken for electrophysiological recording in the vSub-NAc pathway. HFS to the vSub was applied approximately $1.5 \mathrm{~h}$ after the stressor ended. (b) Bilateral intra-BLA RU-38486 - amplitude: When the GR antagonist RU-38486 was microinjected bilaterally into the BLA, the Stress-Vehicle group showed reduced amplitude levels compared with the No StressVehicle and Stress-RU groups. The No Stress-RU group showed reduced amplitude levels compared with the No Stress-Vehicle and the Stress-RU groups $(* P<0.05$; $* * P<0.0$ I; ***P $<0.00$ I). (c) Bilateral intra-BLA RU-38486 — slope: When the GR antagonist RU-38486 was microinjected bilaterally into the BLA the Stress-Vehicle group showed reduced slope levels compared with the No Stress-Vehicle and Stress-RU groups. The No Stress-RU group showed reduced amplitude levels compared with the Stress-RU group $(* P<0.05$; $* * P<0.0 I)$. (d) Input-output curve: No significant differences between the groups were found in NAc EFP amplitudes after stimulation of vSub input with different stimulus intensities. (e) Bilateral intra-BLA WIN55,2 I 2-2 - amplitude: When the $\mathrm{CBI} / 2$ agonist WIN55,2I 2-2 was microinjected bilaterally into the BLA the Stress-Vehicle group showed reduced amplitude levels compared with the StressWIN and the No Stress-Vehicle groups (*** $P<0.00$ I). (f) Bilateral intra-BLA WIN55,2I2-2 - slope: When the CBI/2 agonist WIN55,2I2-2 was microinjected bilaterally into the BLA the Stress-Vehicle group showed reduced slope levels compared with the No Stress-Vehicle and Stress-WIN groups $(* P=0.05$; **P $<0.0 \mathrm{I})$. (g) Input-output curve: No significant differences between the groups were found in NAc EFP amplitudes after stimulation of vSub input with different stimulus intensities. (h) Intra-BLA AM25 I - amplitude: When RU 38486 or WIN55,2I2-2 were co-administered into the BLA with the $\mathrm{CBI}$ receptor antagonist $\mathrm{AM} 25 \mathrm{I}$ the Stress-AM+WIN group showed reduced amplitude levels compared with the other groups (*P $<0.05$ ). (i) Intra-BLA AM25 I - slope: When RU 38486 or WIN55,2 I2-2 were co-administered into the BLA with the CBI receptor antagonist AM25 I the Stress-AM+WIN group showed reduced amplitude levels compared with the No Stress-AM group $(* P<0.05)$.
} 
a

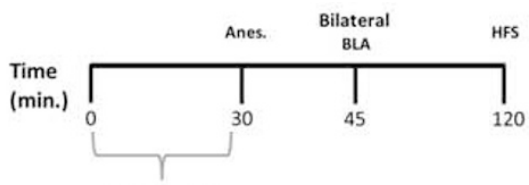

EP/No EP

b

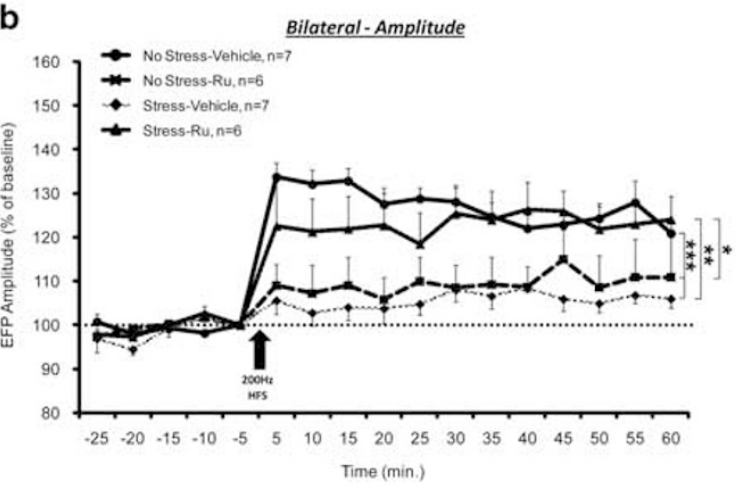

C

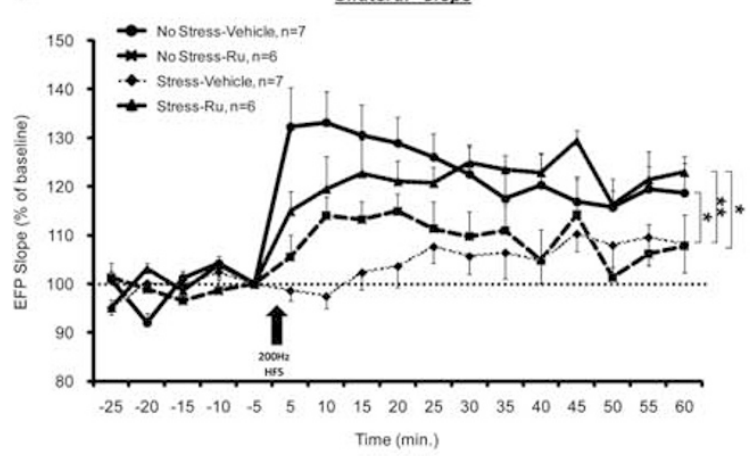

d

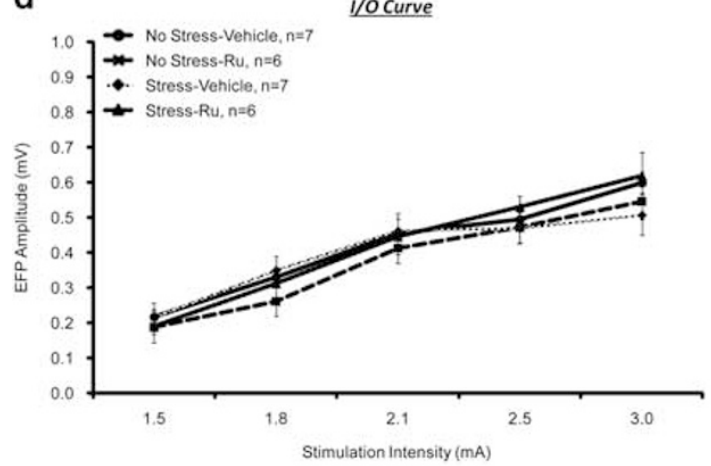

e

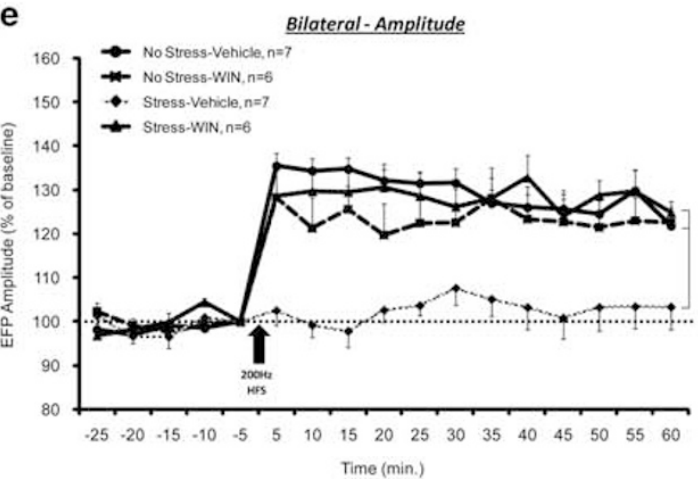

f

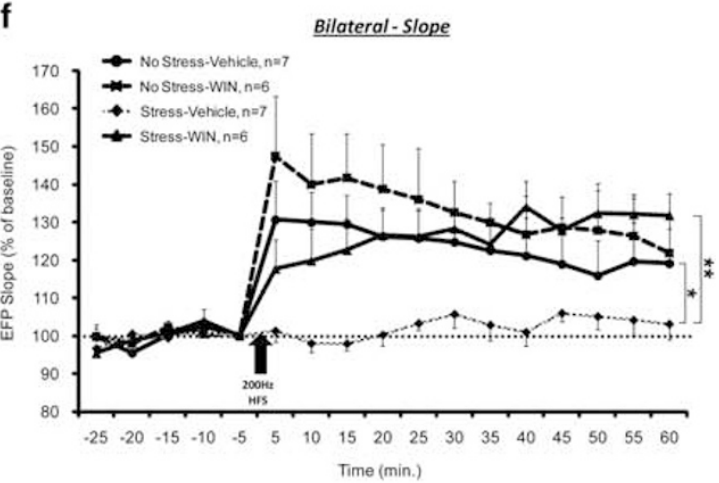

g

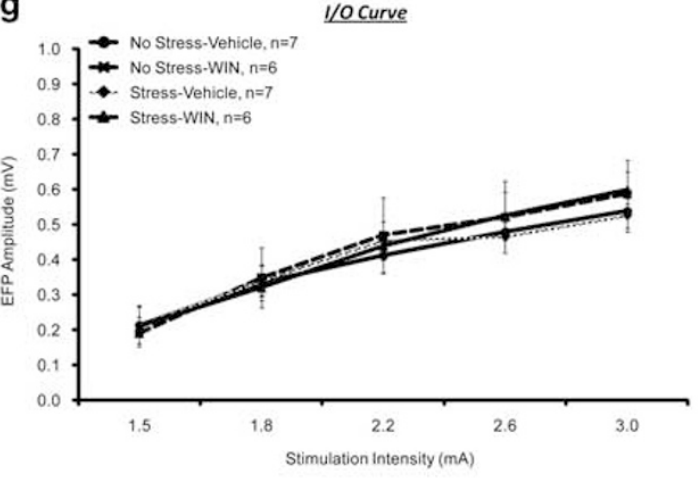

h

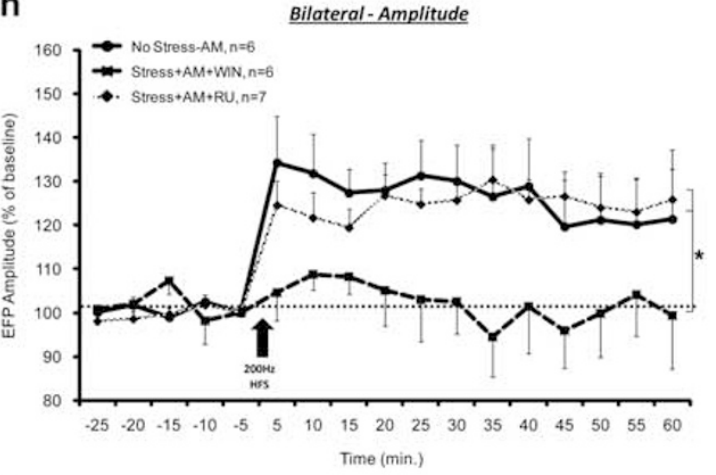

i

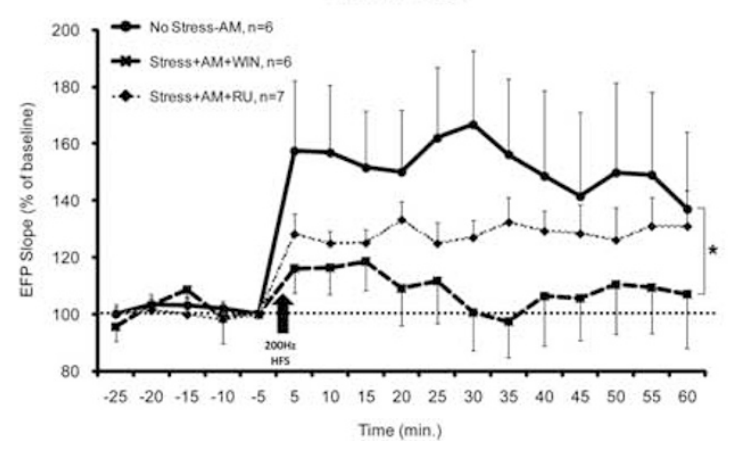


Stress-WIN (amplitude: $P=0.001$; slope: $P<0.01$ ) groups. Hence, bilateral intra-BLA WIN also reversed the stressinduced impairment in LTP.

In Figure $1 \mathrm{c}$ and $\mathrm{f}$, intra-BLA bilateral microinjections of WIN and RU normalized LTP levels, but the levels of slope potentiation in the first 10 min after HFS were lower than observed $1 \mathrm{~h}$ after HFS. These differences between the very early phases after HFS and the later phases may suggest that the bilateral manipulations are not as effective as the ipsilateral or contralateral manipulations in preventing the effects of stress on plasticity. Bilateral microinjections involve a double amount of the drug than the ipsilateral or contralateral microinjections and hence may result in nonspecific effects.

Analysis of the input-output curves revealed a significant effect for stimulation intensity on amplitude (Figure 1g; $\left.\mathrm{F}_{(1,22)}=183.18, P<0.001\right)$. No significant effect on basal EFPs was found.

Glucocorticoids recruit eCB signaling in the BLA and the hippocampus to modulate aversive memory consolidation (Atsak et al, 2012; Campolongo et al, 2009), suggesting that eCBs are located downstream from the GR site of action. Hence, we examined whether the preventing effects of BLA RU and WIN (as WIN is a CB1/CB2 receptor agonist) on plasticity are mediated by the $\mathrm{CB} 1$ receptor by using a combination of WIN/RU and a non-impairing dose of the CB1 receptor antagonist AM251.

Rats were exposed to stress and bilaterally intra-BLA microinjected with AM251+WIN (Stress-AM+WIN, $n=6$ ), AM251+RU (Stress-AM+RU, $n=7$ ), or AM251 with no stress (No Stress-AM, $n=6$ ).

Post-HFS analysis on amplitude (Figure 1h) and slope (Figure 1i) indicated a significant effect for group (amplitude: $\mathrm{F}_{(2,16)}=4.08, \quad P<0.05 ; \quad$ slope: $\mathrm{F}_{(2,16)}=3.52$, $P=0.050)$.

Post-hoc analysis revealed a significant difference between the Stress-AM+WIN group and the No Stress-AM (amplitude: $P<0.05$; slope: $P<0.05$ ) and Stress-AM+RU (amplitude: $P<0.05)$ groups. Co-administering AM251 and WIN, but not RU, into the BLA after stress impaired LTP.

See Figure 2 for histological verification of infusion needle and electrophysiological recording and stimulating sites.

\section{The Effects of Ipsilateral and Contralateral Intra-BLA RU-38486 or WIN55,212-2 on vSub-NAc LTP After Stress}

In order to test whether BLA influences vSub-NAc neuroplasticity after stress directly via anatomical connections, we examined the effects of intra-BLA ipsilateral and contralateral injections. Our working hypothesis was that if the main mode of action of the drugs was via direct anatomical connections, then mostly ipsilateral and not contralateral BLA manipulations would affect LTP (Figure 3a).

In ipsilateral-BLA RU, post-HFS analysis on amplitude (Figure $3 \mathrm{~b}$ ) and slope (Figure $3 \mathrm{c}$ ) indicated significant effects for Drug (amplitude: $\mathrm{F}_{(1,21)}=13.14, \quad P<0.01$; slope: $\mathrm{F}_{(1,21)}=15.33, P=0.001$ ), Stress (amplitude: $\mathrm{F}_{(1,21)}=10.17$, $P<0.01$; slope: $\left.\mathrm{F}_{(1,21)}=13.62, P=0.001\right)$, and Stress $\times$ Drug interaction (amplitude: $\mathrm{F}_{(1,21)}=7.84, P=0.011$ ).

Post-hoc comparison revealed a significant difference between the Stress-Vehicle group and the Stress-RU

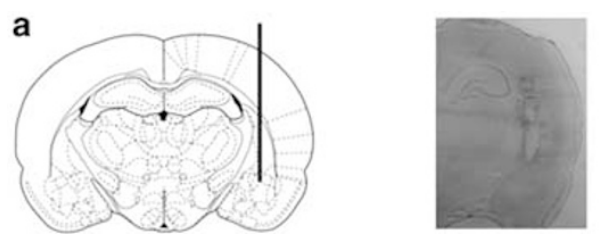

b
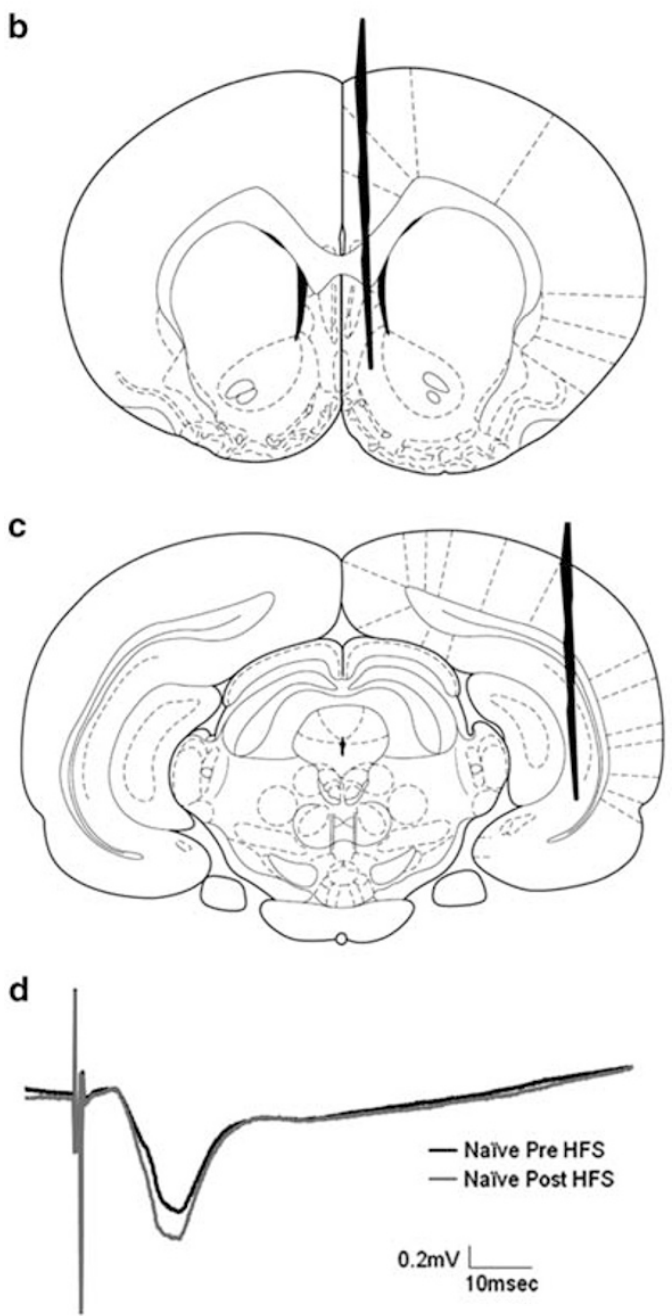

Figure 2 Histological verification of infusion needle placement and electrophysiological recording and stimulating sites. (a) On the left, schematic of coronal sections of the rat brain (Paxinos and Watson, 1997) representing infusion needle sites in the basolateral amygdala. On the right, photomicrograph illustrating a typical cannula track in the BLA (coordinates: $2.7 \mathrm{~mm}$ posterior, $5 \mathrm{~mm}$ lateral, and $6.2 \mathrm{~mm}$ ventral to bregma). (b) Schematic of coronal sections of the rat brain representing recording sites in the nucleus accumbens shell (coordinates: $1.6 \mathrm{~mm}$ anterior, I mm lateral, $5 \mathrm{~mm}$ ventral to bregma). (c) Schematic of coronal sections of the rat brain representing stimulation location of the ventral subiculum (coordinates: $6.5 \mathrm{~mm}$ posterior, $5 \mathrm{~mm}$ lateral, and $6 \mathrm{~mm}$ ventral to bregma). (d) Representative signal trace in the NAc taken before and I $\mathrm{h}$ after HFS to the vSub.

(amplitude and slope: $P<0.01$ ) and No Stress-Vehicle (amplitude and slope: $P<0.001$ ) groups. Hence, ipsilateral intra-BLA RU reversed the stress-induced impairment in LTP.

In ipsilateral-BLA WIN, post-HFS on amplitude (Figure 3d) and slope (Figure 3e) indicated significant effects 
a

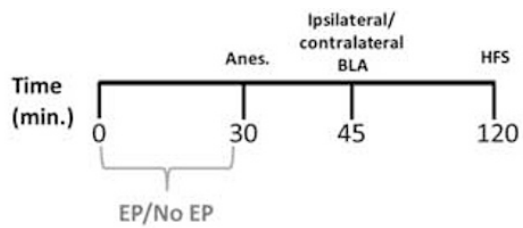

b

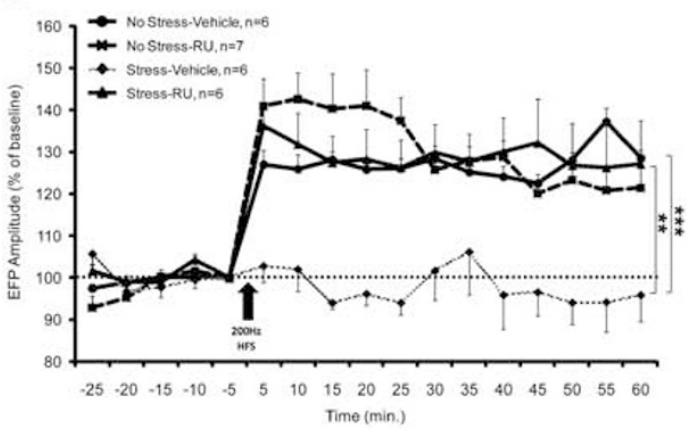

C

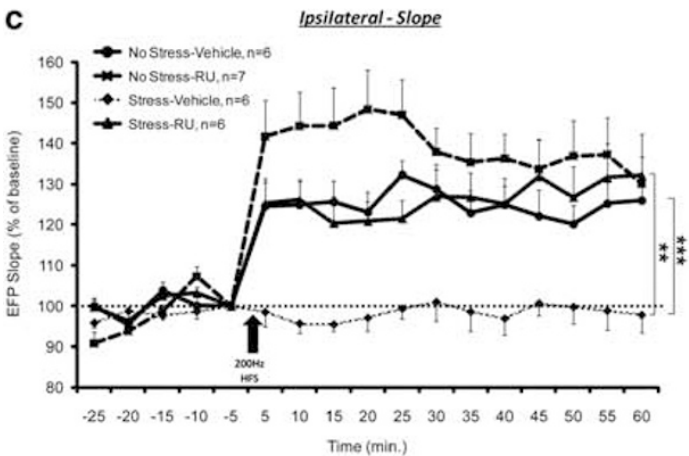

d

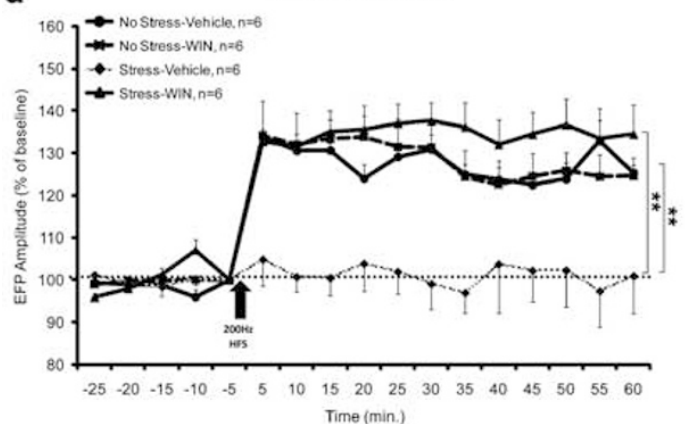

e

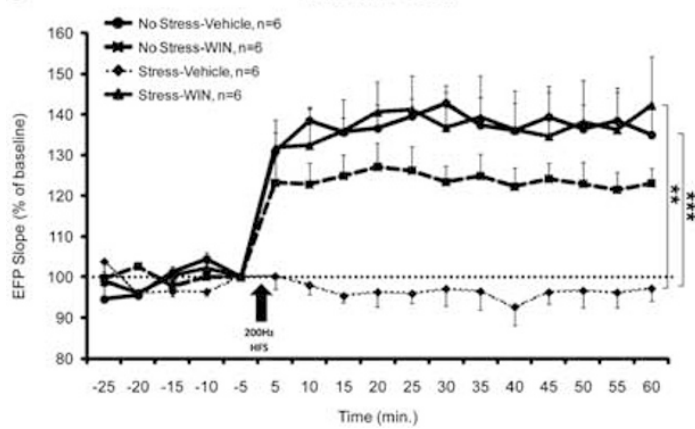

f

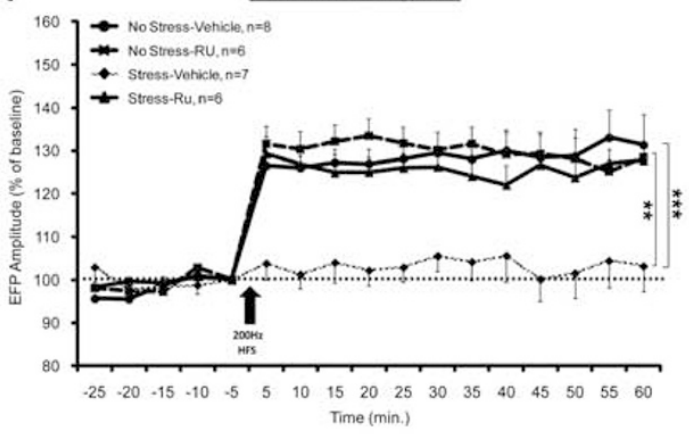

g

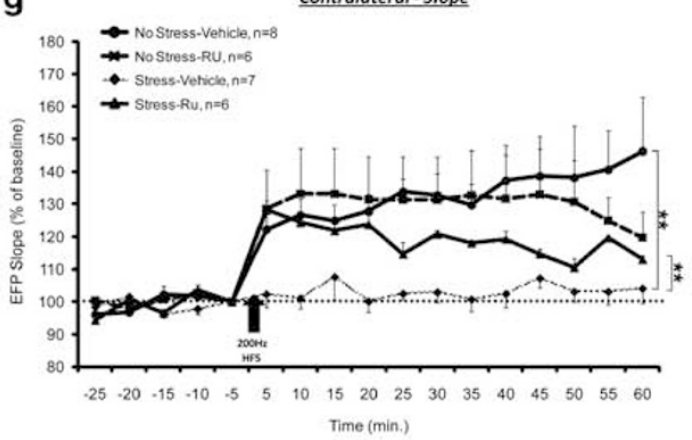

h $\rightarrow$ NoStross:Vehicle, $n=7 \quad$ Contralateral-Amplitude

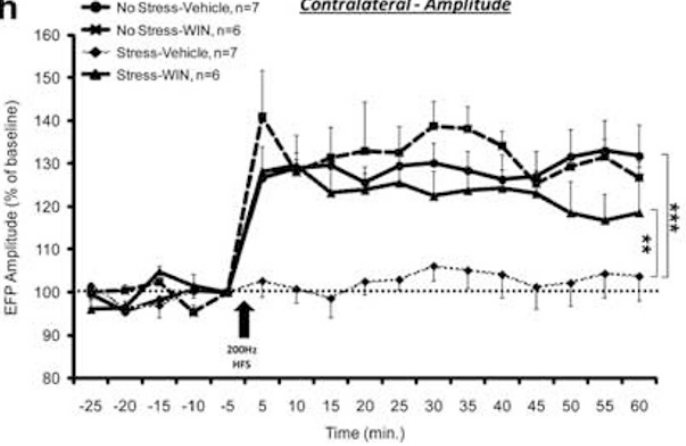

i

Contralateral-Slope

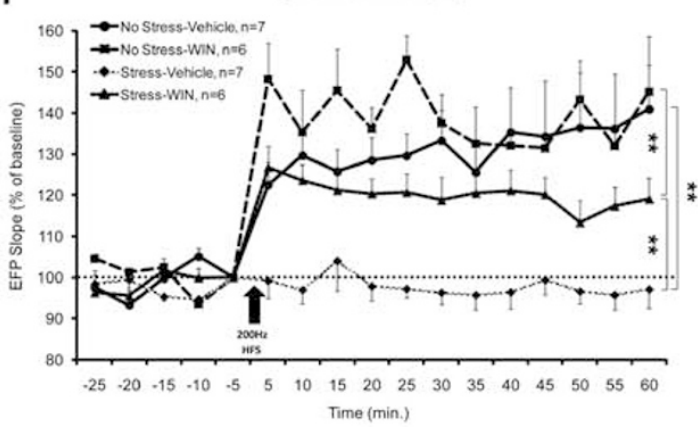

Figure 3 See caption on page 1072. 
for Drug (amplitude: $\mathrm{F}_{(1,20)}=13.43, P<0.01$ ), Stress $\times$ Drug interaction (amplitude: $\mathrm{F}_{(1,20)}=11.42, \quad P<0.01$; slope: $\left.\mathrm{F}_{(1,20)}=17.03, \quad P=0.001\right)$, and Time $\times$ Stress interaction (amplitude: $\mathrm{F}_{(1,20)}=5.51, P<0.05$ ).

Post-hoc comparison revealed a significant difference between the Stress-Vehicle group and the Stress-WIN (amplitude and slope: $P<0.01$ ) and No Stress-Vehicle (amplitude: $P<0.01$; slope: $P<0.001)$ groups. Hence, ipsilateral intra-BLA WIN reversed the stress-induced impairment in LTP.

In contralateral-BLA RU, post-HFS analysis on amplitude (Figure 3f) and slope (Figure 3g) indicated significant main effects for Drug (amplitude: $\mathrm{F}_{(1,23)}=9.1, P<0.01$ ), Stress (amplitude: $\mathrm{F}_{(1,23)}=18.8, \quad P<0.001$; slope: $\mathrm{F}_{(1,23)}=7.37$, $P=0.012$ ), and Time $\times$ Drug interaction (slope: $\mathrm{F}_{(1,23)}=4.43$, $P<0.05)$.

Post-hoc comparison revealed a significant difference between the Stress-Vehicle group and the No StressVehicle (amplitude: $P=0.001$; slope: $P<0.01$ ) and StressRU (amplitude and slope: $P<0.01$ ) groups. These results suggest that contralateral intra-BLA RU reversed this stressinduced impairment in LTP.

In contralateral-BLA WIN, post-HFS analysis on amplitude (Figure $3 \mathrm{~h}$ ) and slope (Figure 3i) indicated significant main effects for Drug (amplitude: $\mathrm{F}_{(1,22)}=4.1, P=0.050$; slope: $\mathrm{F}_{(1,22)}=9.75, \quad P<0.01$ ), and Stress (amplitude $\mathrm{F}_{(1,22)}=10.83, P<0.01$; slope: $\left.\mathrm{F}_{(1,22)}=23.64, P<0.001\right)$.

Post-hoc comparison revealed significant differences between the Stress-Vehicle group and the No Stress-Vehicle (amplitude: $P=0.001$; slope: $P<0.01$ ) and Stress-WIN (amplitude and slope: $P<0.01$ ) groups, and between the No Stress-WIN group and the Stress-WIN group (slope: $P<0.01)$. These results suggest that contralateral intra-BLA WIN reversed the stress-induced impairment in LTP.

\section{The Effects of Ipsilateral and Contralateral BLA Inactivation on vSub-NAc LTP}

Both WIN and RU reversed the effects of stress on plasticity when microinjected into the ipsilateral and contralateral
BLA. In order to compare the contribution of the ipsilateral $v s$ the contralateral BLA to LTP in the vSub-NAc pathway, we conducted another experiment in which we disrupted the normal processing in the BLA by transiently inactivating the ipsilateral or contralateral BLA using a mixture of the GABA-receptor agonists muscimol $\left(\mathrm{GABA}_{\mathrm{A}}\right.$ receptor) and baclofen $\left(\mathrm{GABA}_{\mathrm{B}}\right.$ receptor) before HFS was administered to the vSub (Figure 4a).

Rats were anesthetized and intra-BLA microinjected with baclofen + muscimol, ipsilaterally (Bac+Mus Ipsilateral, $n=7$ ) or contralaterally (Bac+Mus Contralateral, $n=6$ ).

Post-HFS analysis on amplitude (Figure $4 \mathrm{~b}$ ) and slope (Figure 4c) indicated a significant main effect for Group (amplitude: $\quad \mathrm{F}_{(1,11)}=5.79, \quad P<0.05 ; \quad$ slope: $\quad \mathrm{F}_{(1,11)}=8.7$, $P=0.013$ ) and Time (amplitude: $\mathrm{F}_{(1,11)}=16.57, P<0.01$; slope: $\left.\mathrm{F}_{(1,11)}=9.7, P=0.01\right)$. Hence, ipsilateral BLA inactivation impaired LTP compared with contralateral inactivation. Yet, LTP levels in the contralateral group deteriorated over time (amplitude: $\mathrm{F}_{(1,5)}=6.94, P<0.05$; slope: $\mathrm{F}_{(1,5)}=7.83, P<0.05$ ).

Analysis of input-output curve revealed a significant effect for stimulation intensity $\left(\mathrm{F}_{(1,11)}=54.12, P<0.001\right)$. There were no significant effects on basal EFPs (Figure 4d).

Both ipsilateral and contralateral BLA manipulations with RU or WIN prevented the effects of stress on LTP. When the ipsilateral BLA was inactivated, but not the contralateral BLA, no LTP was induced. This corroborates with tracing studies showing no direct contralateral connections between the BLA and NAc (Friedman et al, 2002). Hence, it is possible that BLA manipulations on the contralateral side first influenced the medial PFC (mPFC) and from there may have affected neural plasticity in the vSub-NAc pathway in the other hemisphere. To test this hypothesis, we added a control experiment in which rats were anesthetized and intra-mPFC microinjected with baclofen+muscimol ipsilaterally (Bac+Mus $\mathrm{mPFC}, n=5)$ and compared with vehicletreated rats (Veh mPFC, $n=5$ ).

Post-HFS analysis on amplitude (Figure $4 \mathrm{f}$ ) and slope (Figure 4g) indicated a significant effect for Group (amplitude: $\mathrm{F}_{(1,8)}=24.819, P<0.001$; slope: $\mathrm{F}_{(1,8)}=21.36$, $P<0.01)$, indicating no LTP in the GABA-treated group.

Figure 3 The effects of ipsilateral and contralateral intra-BLA GR deactivation and CBI/2 receptor activation on synaptic plasticity in the vSub-NAc pathway after stress. (a) Rats were exposed to the EP stress for 30 min or not, followed by anesthesia (Anes.) and ipsilateral intra-BLA microinjections (BLA Microinject.) of vehicle, RU, or WIN. Drug microinjection was performed $15 \mathrm{~min}$ after the stressor ended. Rats were then taken for electrophysiological recording in the vSub-NAc pathway. HFS to the vSub was applied approximately $1.5 \mathrm{~h}$ after the stressor ended. Ipsilateral microinjections were to the same hemisphere as the one where electrophysiological recordings were performed, and all groups were comprised equally of left hemisphere and right hemisphere procedures. (b) Ipsilateral intra-BLA RU-38486 — amplitude: When RU 38486 was microinjected into the ipsilateral BLA the Stress-Vehicle group showed reduced amplitude levels compared with the No Stress-Vehicle and Stress-RU groups (**P<0.0 I; ****P<0.00I). (c) Ipsilateral intra-BLA RU-38486 - slope: When RU 38486 was microinjected into the ipsilateral BLA the Stress-Vehicle group showed reduced slope levels compared with the No Stress-Vehicle and Stress-RU groups (**P<0.0I; **** $P<0.00$ I). (d) Ipsilateral intra-BLA WIN55,2I2-2-amplitude: When WIN55,2I2-2 was microinjected into the ipsilateral BLA the Stress-Vehicle group showed reduced amplitude levels compared with the No Stress-Vehicle and Stress-WIN groups (*** $<0.0$ I). (e) Ipsilateral intraBLA WIN55,2 I2-2 - slope: When WIN55,2I2-2 was microinjected into the ipsilateral BLA the Stress-Vehicle group showed reduced slope levels compared with the No Stress-Vehicle and Stress-WIN groups. The Stress-WIN group showed reduced slope levels compared with the No Stress-WIN group (** $P<0.0$ I; **** $P=0.00 \mathrm{I}$ ). (f) Contralateral intra-BLA RU-38486-amplitude: When RU 38486 was microinjected into the contralateral BLA the StressVehicle group showed reduced amplitude levels compared with the No Stress-Vehicle and Stress-RU groups $(* * P<0.0 \mathrm{I}$; **** $\mathrm{P}<0.00 \mathrm{I})$. ( $\mathrm{g})$ Contralateral intra-BLA RU-38486 — slope: When RU 38486 was microinjected into the contralateral BLA the Stress-Vehicle group showed reduced slope levels compared with the No Stress-Vehicle and Stress-RU groups (*** $<0.01$ ). (h) Contralateral intra-BLA WIN55,212-2-amplitude: When WIN55,2I2-2 was microinjected into the contralateral BLA, the Stress-Vehicle group showed reduced amplitude levels compared with the No Stress-Vehicle and Stress-WIN groups (**P<0.0I; ****P<0.00I). (i) Contralateral intra-BLA WIN55,2I2-2_-slope: When WIN55,2I 2-2 was microinjected into the contralateral BLA the Stress-Vehicle group showed reduced slope levels compared with the No Stress-Vehicle and Stress-WIN groups. The Stress-WIN group showed reduced slope levels compared with the No Stress-WIN group $(* * P<0.01)$. 


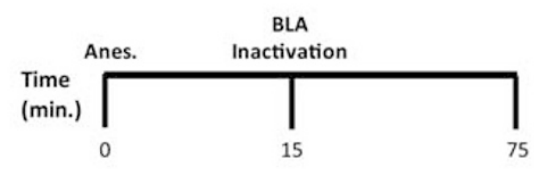

b

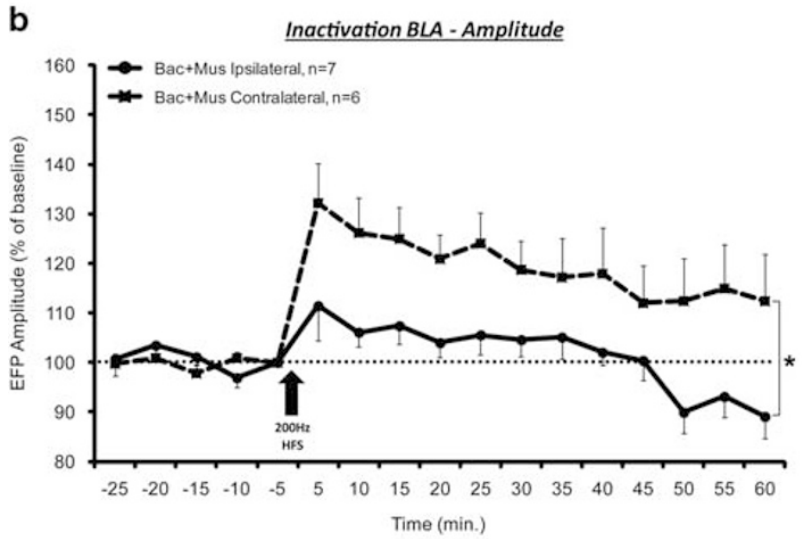

c

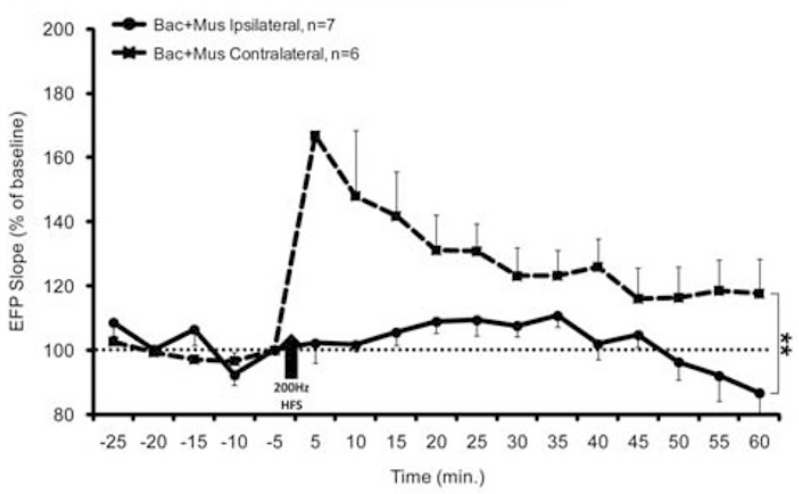

d

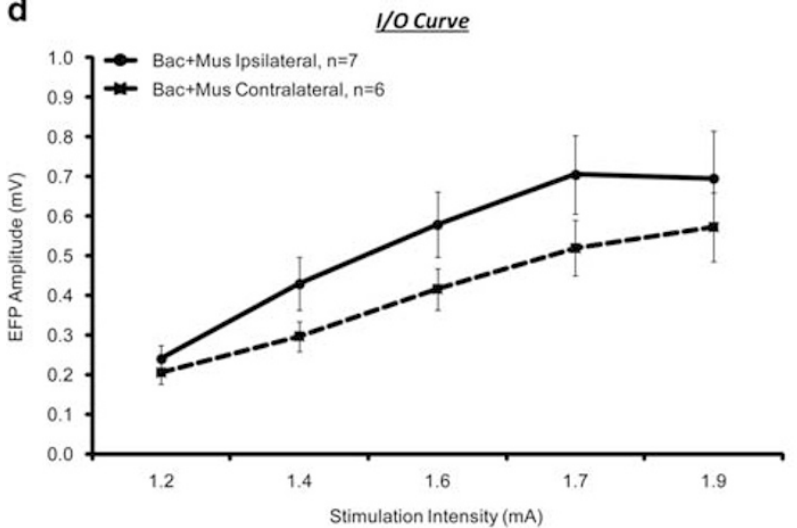

e
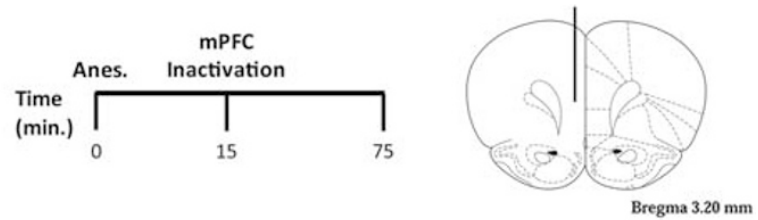

f

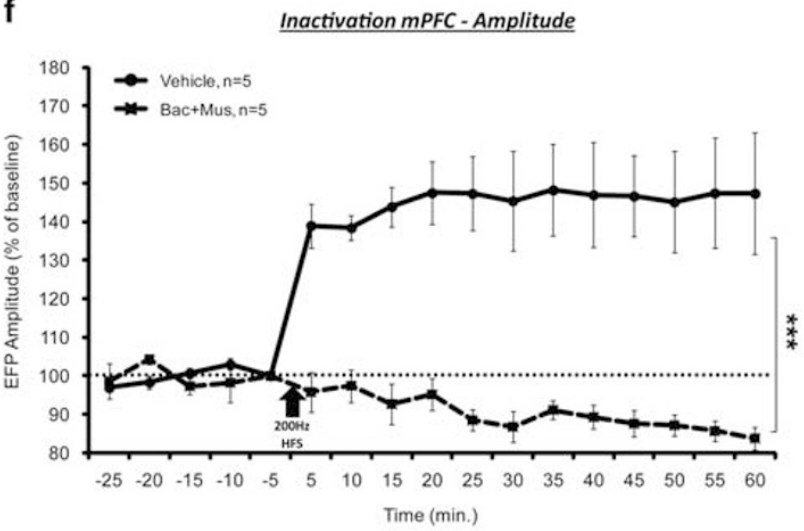

g

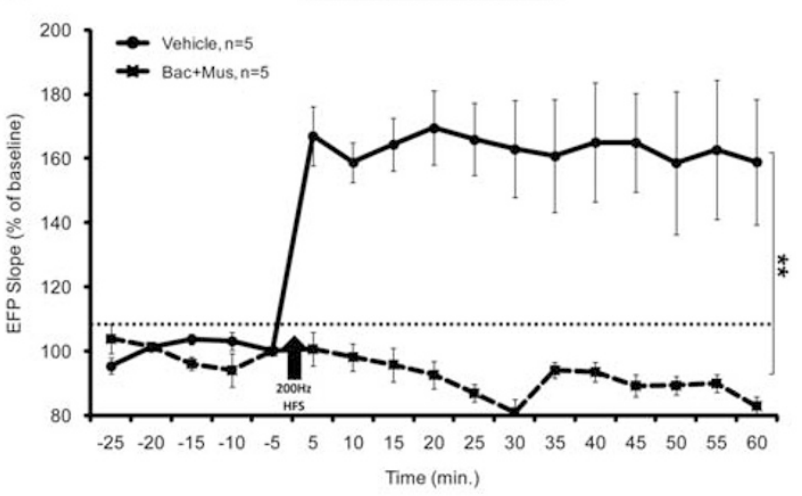

h

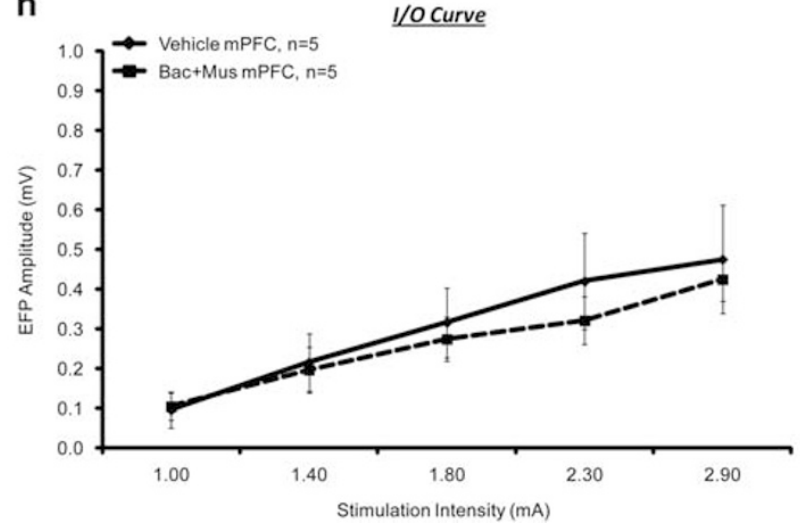

Figure 4 The effects of BLA inactivation on synaptic plasticity in the vSub-NAc pathway. (a) Rats were anesthetized and intra-BLA microinjected with baclofen+muscimol (Bac+Mus), ipsilaterally or contralaterally. They were then taken for electrophysiological recording in the vSub-NAc pathway. HFS to the vSub was applied I h after microinjection. (b) BLA inactivation — amplitude: When GABA agonists were microinjected into the BLA, the Bac+Mus Ipsilateral group showed reduced amplitude levels compared with the Bac+Mus Contralateral group $(* P<0.05)$. (c) BLA inactivation-slope: When GABA agonists were microinjected into the BLA, the Bac+Mus Ipsilateral group showed reduced amplitude levels compared with the Bac+Mus Contralateral group $(* * P=0.01)$. (d) Input-output curve: No significant differences between the groups were found in NAc EFP amplitudes after stimulation of vSub input with different stimulus intensities. (e) Rats were anesthetized and intra-mPFC microinjected with baclofen+muscimol (Bac+Mus mPFC) ipsilaterally or Vehicle. They were then taken for electrophysiological recording in the vSub-NAc pathway. HFS to the vSub was applied I $\mathrm{h}$ after microinjection. On the right, schematic of coronal sections of the rat brain representing infusion needle sites in the medial PFC. (f) mPFC inactivation —amplitude: GABA agonists microinjected into the ipsilateral mPFC demonstrated no LTP compared with the vehicle group (****P<0.00 I). (g) mPFC inactivation-slope: GABA agonists microinjected into the ipsilateral mPFC demonstrated no LTP compared with the vehicle group $(* * P<0.01)$. (h) Input-output curve: No significant differences between the groups were found in NAc EFP amplitudes after stimulation of vSub input with different stimulus intensities. 
Analysis of input-output curve revealed a significant effect for stimulation intensity $\left(\mathrm{F}_{(1,8)}<1\right.$, NS). There were no significant effects on basal EFPs (Figure 4h).

The effects of the different pharmacological treatments on LTP are summarized in Table 1.

\section{The Effects of Intra-BLA RU-38486 or WIN55,212-2 on CREB Activation in the NAc and Amygdala After Stress}

Next we aimed to determine whether intra-BLA RU and WIN can reverse the changes in PCREB after stress exposure (Figure 5a).

We found that total CREB expression was not modulated in the NAc or amygdala in any of the treatment groups. Analysis was performed using two-way ANOVA (Drug $\times$ Stress $(2 \times 2))$.

In the NAc, intra-BLA RU resulted in a significant interaction effect $\left(\mathrm{F}_{(1,28)}=5.94, P<0.05\right)$. Post-hoc comparison revealed significant differences between the Stress-Vehicle group and the Stress-RU $(P<0.05)$ and No Stress-Vehicle $(P<0.05)$ groups (Figure $5 b)$.

In the NAc, intra-BLA WIN resulted in a significant interaction effect $\quad\left(\mathrm{F}_{(1,24)}=7.96, \quad P<0.01\right)$. Post-hoc comparison revealed significant differences between the Stress-Vehicle and the Stress-WIN $(P<0.05)$ and No Stress-Vehicle $(P=0.05)$ groups (Figure $5 c)$. The NAc was obtained by punches bilaterally (Figure $5 \mathrm{~d}$ ).

As previous studies found that stress had no effect on pCREB levels in the MeA (Reagan et al, 2007), we added the $\mathrm{MeA}$ as a control group.

In the MeA, intra-BLA RU did not result in significant effects (Drug: $\mathrm{F}_{(1,29)}<1$, NS; Stress: $\mathrm{F}_{(1,29)}<1$, NS; Drug $\times$ Stress: $\mathrm{F}_{(1,29)}<1$, NS; Figure $\left.5 f\right)$.

In the MeA, intra-BLA WIN resulted in a significant effect for Drug $\left(\mathrm{F}_{(1,32)}=15.06, P<0.001\right)$. Post-hoc comparison revealed a significant difference between the No StressVehicle group and the No Stress-WIN group $(P<0.01)$, and between the Stress-Vehicle group and the Stress-WIN group $(P<0.05)$ (Figure $5 \mathrm{~g})$. Hence, stress had no effect on pCREB levels in the MeA and intra-BLA WIN, regardless of stress exposure, significantly increased MeA pCREB levels.

It has been shown that stress or corticosterone increased pCREB immunoreactivity in the BLA (Roozendaal et al, 2006). Hence, to verify that exposure to the EP stress indeed activated the BLA, we measured PCREB in the BLA after stress exposure. As cannulae were inserted into the BLA and this could affect pCREB levels by itself, we used noncannulated rats that were exposed to the EP stress (Stress) or not (No Stress). We found that total CREB expression was not modulated in the BLA following stress. Independentsamples $t$-test revealed a significant effect for Stress $\left(t_{(10)}=-2.17, P=0.05\right)$, suggesting that exposure to EP stress resulted in significantly higher levels of pCREB compared with rats that were not exposed to stress (Figure 5h). The $\mathrm{MeA}$ and BLA were obtained by punches bilaterally (Figure 5i).

To further support that stress makes the amygdala hyperactive and the accumbens hypoactive, we examined the effects of exposure to the EP stress on neuronal activation in the BLA and NAc using cFOS. Independent-samples $t$-test revealed a significant effect for Stress on cFOS mRNA
Table I Summary of LTP Results

\begin{tabular}{ll}
\hline Impaired vSub-NAc LTP & Intact vSub-NAc LTP \\
\hline Stress & Stress+bilateral intra-BLA RU \\
Bilateral intra-BLA RU (no stress) & Bilateral intra-BLA WIN (no stress) \\
Stress+bilateral AM25 I and WIN & Stress+bilateral intra-BLA WIN \\
Ipsilateral BLA inactivation & Stress+bilateral AM25I and RU \\
Ipsilateral mPFC inactivation & Stress+ipsilateral intra-BLA RU \\
& Stress+ipsilateral intra-BLA WIN \\
& Stress+contralateral intra-BLA RU \\
& Stress+contralateral intra-BLA WIN \\
& Contralateral BLA inactivation \\
\hline
\end{tabular}

Abbreviations: BLA, basolateral amygdala; LTP, long-term potentiation; NAc, nucleus accumbens; RU, RU-38486; vSub, ventral subiculum; WIN, WIN55,2 I2-2. A summary of the effects of stress and pharmacological manipulations on LTP in the vSub-Nac pathway.

expression in the NAc $\left(t_{(14)}=-2.842, P=0.013\right.$; Figure 5e) and BLA $\left(t_{(14)}=2.736, P=0.017\right.$; Figure $\left.5 j\right)$.

\section{DISCUSSION}

The main findings of this study are that acute stress exposure impaired plasticity and inhibited CREB activity in the NAC and that intra-BLA CB1 activation and GR deactivation reversed these stress-induced alterations.

The reversal effects of intra-BLA WIN and RU are in line with behavioral data showing that disruption of GR activity or CB1 enhancement within the BLA can prevent stressinduced effects on learning and memory in tasks involving other brain regions (Ganon-Elazar and Akirav, 2009; Ramot and Akirav, 2012; Roozendaal et al, 2002; Segev et al, 2012, 2014).

Several lines of research suggest that memory-modulatory information from the BLA and the hippocampus converge in the NAc: BLA and hippocampal information converge to single cells in the NAc (O'Donnell and Grace, 1995), BLA stimulation prior to hippocampal stimulation increased the probability that hippocampal stimulation would evoke NAc neurons firing (Mulder et al, 1998), an intact BLA-NAc pathway is critical for the enhancing effects of glucocorticoids on hippocampal memory consolidation (Setlow et al, 2000), and disruption of the BLA-NAc pathway blocks the enhancing effects of intrahippocampal GR agonist infusions on memory consolidation (Roozendaal et al, 2001).

Gill and Grace (2011) demonstrated that BLA hyperactivation using theta burst stimulation disrupted the normal processing within the NAc and suppressed vSub-evoked responses in the NAc. They also showed no potentiation in the vSub-NAc pathway following HFS to rats exposed to repeated restraint (Gill and Grace, 2013). Similarly, we show that exposure to stress increased cFOS mRNA and pCREB levels in the BLA, suggesting enhanced neuronal activation while decreasing cFOS and pCREB in the NAc, suggesting hypoactivation. The same stressor also impaired plasticity in the vSub-NAc pathway. 
Together with results showing a decrease in pCREB in the NAc after some stressors (our study and Barrot et al, 2005) and an increase in PCREB in the NAc following different stressors (Pliakas et al, 2001; Barrot et al, 2002), we can conclude that stress disrupts NAc functionality, but the direction of disruption is dependent on the specific stressor under examination. Importantly, intra-BLA RU and WIN reversed the stress-induced decrease in NAc pCREB, similar to the reversal effects of antidepressants on CREB activity (Wallace et al, 2009).

A non-impairing dose of intra-BLA AM251 had no effect on LTP levels similar to previous behavioral findings (Campolongo et al, 2009; Ganon-Elazar and Akirav, 2013). Co-administering AM251 with WIN or RU showed that BLA $\mathrm{CB} 1$ receptors are required for the preventing effects of WIN after stress but are not required for RU to exert a protective effect on NAc plasticity. This corroborates other studies suggesting that the eCBs are located downstream from the GR site of action (Atsak et al, 2015).

Intra-BLA RU with no stress exposure impaired LTP when microinjected bilaterally but not when microinjected ipsilaterally or contralaterally. Other studies showed that intra-BLA RU modulates learning and memory (Campolongo et al, 2009; Segev and Akirav, 2011). Possibly, optimal BLA glucocorticoid levels are required for LTP in the vSub-NAc pathway. Rats with bilateral microinjections received a double amount of each drug. Hence, bilateral intra-BLA RU, with no stress exposure, possibly reduced glucocorticoid levels below optimal levels and impaired LTP. When rats were exposed to stress, which significantly elevates glucocorticoid levels, LTP was impaired. However, stress exposure followed by BLA GR blockade may have restored glucocorticoids to optimal levels enabling LTP.

Furthermore, we found differences in the Stress-WIN and Stress-RU bilateral groups between the very early phases after HFS and the later phases. There were no such effects in the ipsilateral or contralateral groups, further suggesting that the effects of the bilateral manipulations are not as robust as the ipsilateral or contralateral manipulations; this could be due to a double amount of the drugs that may result in nonspecific effects, such as reduced levels of posttetanic potentiation (PTP; the enhancement in EFPs observed shortly after the induction of HFS).

It has been suggested that the rapid effects of glucocorticoids on memory are mediated in a non-genomic manner, via a membrane-bound receptor (Barsegyan et al, 2010; Tasker et al, 2006). RU was shown to block activation of the membrane-bound GR by a membrane impermeable conjugate of corticosterone (Barsegyan et al, 2010; Karst et al, 2010; Roozendaal et al, 2010). Roozendaal et al (2010) suggested that activation of the membrane-bound GR can result in increased pCREB, therefore it is plausible that the effects of RU on LTP and pCREB levels were also mediated by non-genomic mechanisms especially as microinjection occurred shortly after acute stress exposure.

We found that both ipsilateral and contralateral intra-BLA WIN and RU reversed the stress-induced impairment in plasticity. To differentiate between ipsilateral and contralateral contribution to LTP, we inactivated the ipsilateral or contralateral BLA using GABA agonists. Inactivating the ipsilateral BLA prevented LTP induction, whereas inactivating the contralateral BLA resulted in significant potentiation that decreased over time, but still stable LTP was maintained. Hence, the ipsilateral effects are likely mediated through direct BLA monosynaptic connections to the NAc, whereas the contralateral effects are mediated through indirect network projections.

Both BLA hyperactivation (ie, using stress exposure) and BLA inactivation resulted in no LTP in the vSub-NAc pathway. A support for the inactivation results comes from studies demonstrating that BLA lesions block the effects of posttraining intra-hippocampal glucocorticoid administration on memory for several tasks (Roozendaal and McGaugh, 1996). Specifically, an intact BLA-NAc pathway is critical for the enhancing effects of glucocorticoids on memory consolidation of an inhibitory avoidance task, which depends on the hippocampus, BLA, and NAc (Setlow et al, 2000). BLA hyperactivation also impairs LTP; BLA theta burst stimulation or exposure to repeated restraint suppressed vSub-NAc evoked responses (Gill and Grace, 2011, 2013).

The BLA may influence stress effects on vSub-NAc neuroplasticity directly via anatomical connections. If the anatomical pathway is the main mode of effect, then we would expect that mostly ipsilateral and not contralateral BLA manipulations would affect vSub-NAc neuroplasticity. Although both ipsilateral and contralateral BLA manipulations prevented the effects of stress, there were differential effects on LTP when the BLA was inactivated; when the ipsilateral BLA was inactivated, but not when the contralateral BLA was inactivated, no LTP was induced. As tracing studies show no direct contralateral connections between the BLA and NAc (Friedman et al, 2002), it is possible that BLA manipulations on the contralateral side first influenced the $\mathrm{mPFC}$ and from there may have affected neural plasticity in the other hemisphere. In support, we found that inactivating the ipsilateral mPFC using GABA agonists blocked the ability to induce LTP in the NAc following HFS to the vSub. Hence, this supports the possibility that the contralateral effects of the BLA on the NAc are mediated via the mPFC.

Several lines of evidence seem to support the anatomical explanation. There are reciprocal connections between the $\mathrm{mPFC}$ and BLA that regulate affect and memory (Garcia et al, 1999; Laviolette and Grace, 2006); mPFC activity is known to constrain BLA activity, whereas stress and glucocorticoids alter $\mathrm{mPFC}$ functioning thereby increasing BLA responses to emotionally arousing stimuli (Lyons et al, 2000; Amat et al, 2005); not only the mPFC and vSub compete for the control of NAc neurons after tetanic stimulation (Goto and Grace, 2005) but also the initiation of vSub drive of the NAc requires a contributory role of the mPFC (Belujon and Grace, 2008). At the earliest stage of activation, the mPFC must exert a permissive action to allow the vSub to control the NAc, indicating a dependence on the $\mathrm{mPFC}$ of the vSub drive of NAc neurons (Belujon and Grace, 2008). However, in the case where the vSub-NAc drive is sufficient to result in the induction of LTP, the participation of the $\mathrm{mPFC}$ is no longer required to enable vSubNAc drive.

Taken together, the results suggest that the BLA and the mPFC modulate plasticity in the vSub-NAc pathway. Manipulating the ipsilateral or contralateral BLA with RU or WIN prevented the stress-induced impairment of vSubNAc LTP, but contralateral inactivation of the BLA did not 
a

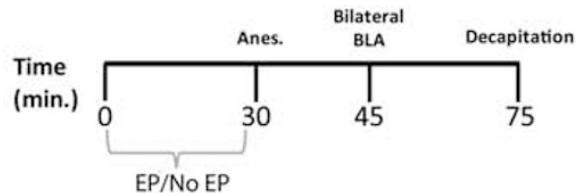

b
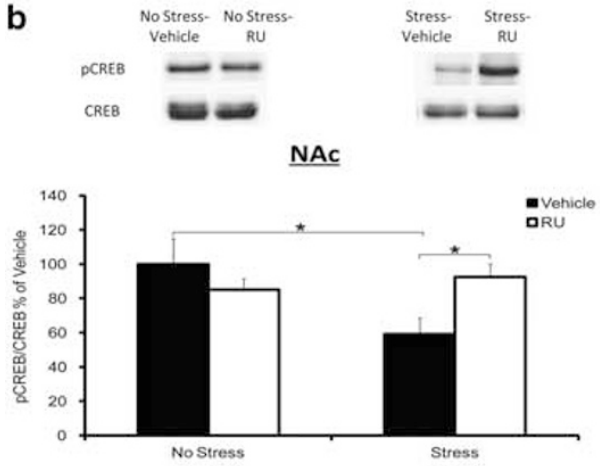

C
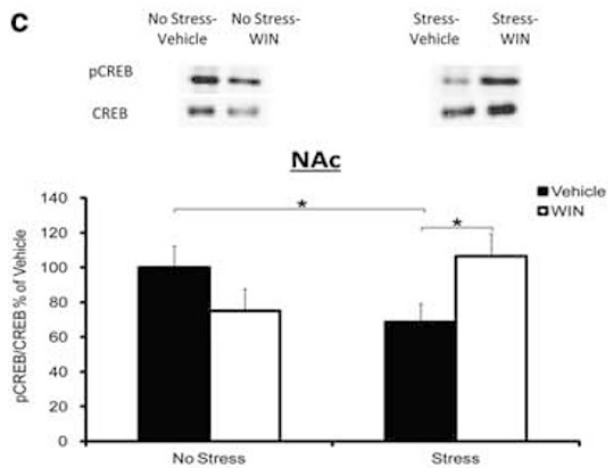

d

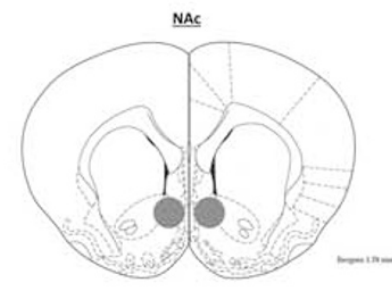

e

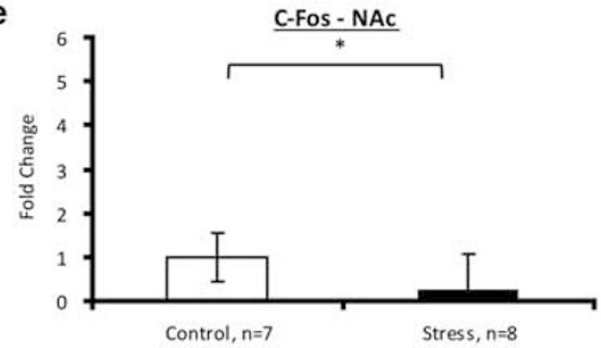

f
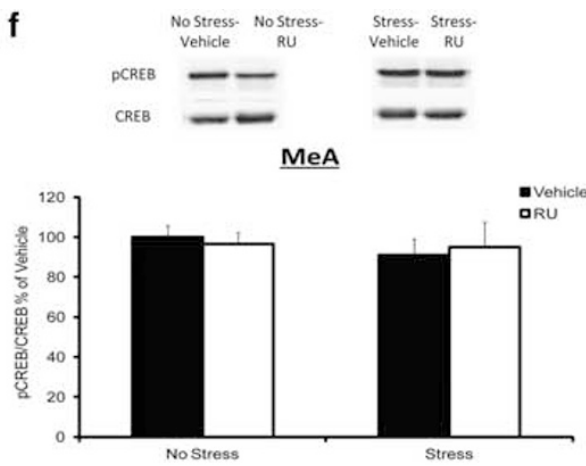

g

Stress

No Stress- No Stress- Stress- Stress-

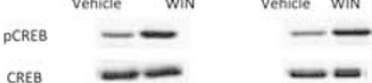

CREB $\quad-0$

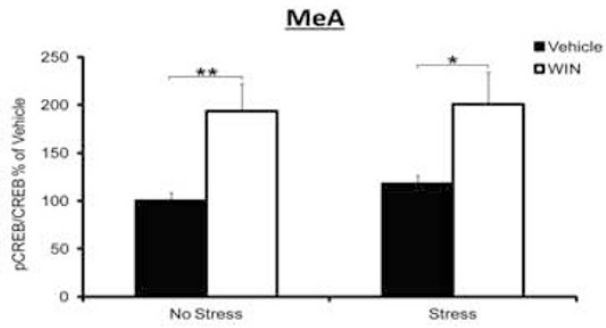

h

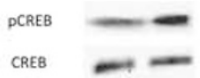

BLA-Not cannulated

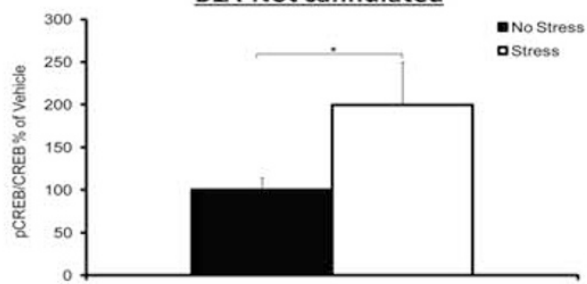

i

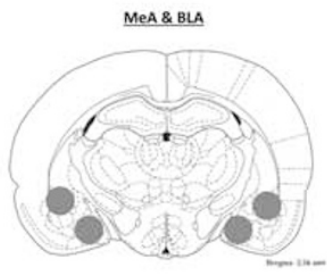

i

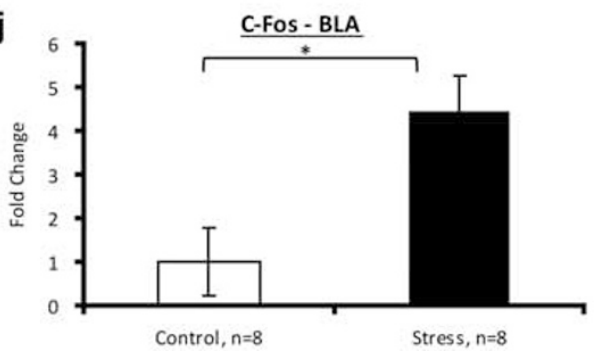


Figure 5 The effects of stress and intra-BLA RU or WIN55,2 I2-2 on CREB activity. (a) Rats were exposed to the EP stress for 30 minutes or not, followed by anesthesia and microinjected bilaterally into the BLA with vehicle, RU, or WIN. After 30 minutes, the rats were killed and their brains were frozen at $-80^{\circ} \mathrm{C}$ for western blot analysis of CREB and pCREB. (b) In the NAc, the Stress-Vehicle $(n=9)$ group showed reduced CREB activation levels compared to the Stress-RU ( $n=7)$ and No Stress-Vehicle $(n=9)$ groups (*P<0.05; RU: $n=8)$. (c) In the NAc, the Stress-Vehicle ( $n=7)$ group showed reduced CREB activation levels compared to the Stress-WIN $(n=6)$ and No Stress-Vehicle $(n=5)$ groups (* $P=0.05 ; \mathrm{WIN}: n=5)$. (d) Brain sites from where the tissue samples were extracted. The nucleus accumbens (NAc) shell was obtained by punches ( $1.0 \mathrm{~mm}$ diameter) bilaterally. The numbers refer to the distance from Bregma (based on Paxinos and Watson, 1997). (e) cFOS mRNA expression is decreased in the NAc after exposure to the EP stress (*P<0.05). (f) Intra-BLA RU did not result in any significant differences between the groups in pCREB in the MeA (Vehicle: $n=8$; Stress-Vehicle: $n=9$; RU: $n=7$; Stress RU=9). (g) Intra-BLA WIN increased pCREB levels in the MeA $(* P<0.05 ; * * P<0.01 ; n=7$ in all experimental groups). ( $h$ ) In the BLA, the No Stress group showed reduced $p C R E B$ levels compared to the Stress group (* $P=0.05$ ). (i) Brain sites from where the tissue samples were extracted. The medial amygdala (MeA) and basolateral amygdala (BLA) were obtained by punches ( $1.0 \mathrm{~mm}$ diameter) bilaterally. The number refers to the distance from Bregma (based on Paxinos and Watson, 1997). (j) cFOS mRNA expression is increased in the BLA after exposure to the EP stress $(* P<0.05)$.

block plasticity. Hence, the ipsilateral effects are likely mediated through direct monosynaptic connections to the accumbens, whereas the stress effects and RU/WIN effects seem to be mediated through indirect network projections. The findings strongly support in favor of an anatomical pathway from the BLA (perhaps via the mPFC) that mediates the effects on vSub-NAc plasticity.

Nevertheless, other alternative peripheral pathways cannot be ruled out. BLA manipulations may be mediated by HPA axis and noradrenergic or dopaminergic systems. Cannabinoid activation and GR blockade reduce stress-induced HPA axis activation through GABAergic mechanism in the BLA (Akirav, 2013; Ganon-Elazar and Akirav, 2009, 2012, 2013; Hill et al, 2007, 2009; Rademacher et al, 2008; Segev et al, 2014). The noradrenergic antagonist propranolol blocked the effects of WIN on memory, indicating that eCBs enhance the consolidation of memory via an interaction with the noradrenergic arousal system (Campolongo et al, 2013; Atsak et al, 2015). Finally, the release of dopamine is enhanced in NAc shell during exploration of a novel environment (Rebec et al, 1997), and acute stress led to a low frequency stimulation-induced potentiation of vSub input to the NAc that was mediated by $\mathrm{D}_{2}$ receptors (Gill and Grace, 2013).

\section{Summary}

As a stressful life experience is a key predisposing factor to the development of drug addiction as well as other forms of psychiatric disease, and particularly depression that is associated with neuroplastic changes in the NAc (Bessa et al, 2013), the influence of stressful events on synaptic plasticity and CREB activity in the NAc may aid in understanding the neurobiological mechanisms underlying behavioral pathology caused by stress.

Neuropsychiatric disorders such as depression are conceptualized as an inability of neuronal systems to adequately adapt to aversive stimuli such as stress, while antidepressants exert their effect through the reconstitution or enhancement of neuronal plasticity (Duman et al, 1999). Our findings demonstrate that neuronal activity and plasticity in the NAc are significantly altered by stress and suggest that the eCB and glucocorticoid systems in the BLA can restore normal accumbal function and hence may have a central role in the treatment of a variety of stress-related disorders.

\section{FUNDING AND DISCLOSURE}

The authors declare no conflict of interest.

\section{ACKNOWLEDGMENTS}

This research was supported by THE ISRAEL SCIENCE FOUNDATION (grant No. 222/08 to IA) (http://www.isf. org.il/). We thank Tomer Mizrachi Zer-Aviv, Nachshon Korem, and Salman Zubidat for their valuable help.

\section{REFERENCES}

Abush H, Akirav I (2013). Cannabinoids ameliorate impairments induced by chronic stress to synaptic plasticity and shortterm memory. Neuropsychopharmacology 38: 1521-1534.

Akirav I (2013). Cannabinoids and glucocorticoids modulate emotional memory after stress. Neurosci Biobehav Rev 7: 2554-2563.

Amat J, Baratta MW, Paul E, Bland ST, Watkins LR, Maier SF (2005). Medial prefrontal cortex determines how stressor controllability affects behavior and dorsal raphe nucleus. Nat Neurosci 8: 365-371.

Atsak P, Hauer D, Campolongo P, Schelling G, Fornari RV, Roozendaal B (2015). Endocannabinoid signaling within the basolateral amygdala integrates multiple stress hormone effects on memory consolidation. Neuropsychopharmacology 40: 1485-1494.

Atsak P, Hauer D, Campolongo P, Schelling G, McGaugh JL, Roozendaal B (2012). Glucocorticoids interact with the hippocampal endocannabinoid system in impairing retrieval of contextual fear memory. PNAS 109: 3504-3509.

Barrot M, Olivier JDA, Perrotti LI, DiLeone RJ, Berton O, Eisch AJ et al (2002). CREB activity in the nucleus accumbens shell controls gating of behavioral responses to emotional stimuli. PNAS 99: 11435-11440.

Barrot M, Wallace DL, Bolaños CA, Graham DL, Perrotti LI, Neve RL et al (2005). Regulation of anxiety and initiation of sexual behavior by CREB in the nucleus accumbens. PNAS 102: 8357-8362.

Barsegyan A, Mackenzie SM, Kurose BD, McGaugh JL, Roozendaal B (2010). Glucocorticoids in the prefrontal cortex enhance memory consolidation and impair working memory by a common neural mechanism. PNAS 107: 16655-16660.

Belujon P, Grace AA (2008). Critical role of the prefrontal cortex in the regulation of hippocampus-accumbens information flow. J Neurosci 28: 9797-9805.

Bessa JM, Morais M, Marques F, Pinto L, Palha JA, Almeida OFX et al (2013). Stress-induced anhedonia is associated with hypertrophy of medium spiny neurons of the nucleus accumbens. Transl Psychiatry 3: e266.

Bourtchuladze R, Frenguelli B, Blendy J, Cioffi D, Schutz G, Silva AJ (1994). Deficient long-term memory in mice with a targeted mutation of the cAMP-responsive element-binding protein. Cell 79: 59-68.

Campolongo P, Morena M, Scaccianoce S, Trezza V, Chiarotti F, Schelling G et al (2013). Novelty-induced emotional arousal modulates cannabinoid effects on recognition memory and adrenocortical activity. Neuropsychopharmacology 38: 1276-1286. 
Campolongo P, Roozendaal B, Trezza V, Hauer D, Schelling G, McGaugh JL et al (2009). Endocannabinoids in the rat basolateral amygdala enhance memory consolidation and enable glucocorticoid modulation of memory. PNAS 106: 4888-4893.

Duman RS, Malberg J, Thome J (1999). Neural plasticity to stress and antidepressant treatment. Biol Psychiatry 46: 1181-1191.

Friedman DP, Aggleton JP, Saunders RC (2002). Comparison of hippocampal, amygdala, and perirhinal projections to the nucleus accumbens: combined anterograde and retrograde tracing study in the Macaque brain. J Comp Neurol 450: 345-365.

Ganon-Elazar E, Akirav I (2009). Cannabinoid receptor activation in the basolateral amygdala blocks the effects of stress on the conditioning and extinction of inhibitory avoidance. J Neurosci 29: 11078-11088.

Ganon-Elazar E, Akirav I (2012). Cannabinoids prevent the development of behavioral and endocrine alterations in a rat model of intense stress. Neuropsychopharmacology 37: 456-466.

Ganon-Elazar E, Akirav I (2013). Cannabinoids and traumatic stress modulation of contextual fear extinction and GR expression in the amygdala-hippocampal-prefrontal circuit. Psychoneuroendocrinology 38: 1675-1687.

Garcia R, Volumba RM, Baudry M, Thompson RF (1999). The amygdala modulates prefrontal cortex activity relative to conditioned fear. Nature 402: 294-296.

Gill KM, Grace AA (2011). Heterogeneous processing of amygdala and hippocampal inputs in the rostral and caudal subregions of the nucleus accumbens. Int J Neuropsychopharmacol 14: 1301-1314.

Gill KM, Grace AA (2013). Differential effects of acute and repeated stress on hippocampus and amygdala inputs to the nucleus accumbens shell. Int J Neuropsychopharmacol 6: 2013-2025.

Gordon GJ, Jensen RV, Hsiao LL, Gullans SR, Blumenstock JE, Richards WG et al (2003). Using gene expression ratios to predict outcome among patients with mesothelioma. J Natl Cancer Inst 95: 598-605.

Goto Y, Grace AA (2005). Dopamine-dependent interactions between limbic and prefrontal cortical plasticity in the nucleus accumbens: disruption by cocaine sensitization. Neuron 47: 255-266.

Hill MN, Barr AM, Ho WS, Carrier EJ, Gorzalka BB, Hillard CJ (2007). Electroconvulsive shock treatment differentially modulates cortical and subcortical endocannabinoid activity. $J \mathrm{Neu}$ rochem 103: 47-56.

Hill MN, McLaughlin RJ, Morrish AC, Viau V, Floresco SB, Hillard CJ et al (2009). Suppression of amygdalar endocannabinoid signaling by stress contributes to activation of the hypothalamic-pituitaryadrenal Axis. Neuropsychopharmacology 34: 2733-2745.

Hill MN, McLaughlin RJ, Pan B, Fitzgerald ML, Roberts CJ, Lee TT-Y et al (2011). Recruitment of prefrontal cortical endocannabinoid signaling by glucocorticoids contributes to termination of the stress response. J Neurosci 31: 10506-10515.

Karst H, Berger S, Erdmann G, Schütz G, Joëls M (2010). Metaplasticity of amygdalar responses to the stress hormone corticosterone. PNAS 107: 14449-14454.

Korem N, Akirav I (2014). Cannabinoids prevent the effects of a footshock followed by situational reminders on emotional processing. Neuropsychopharmacology 39: 2709-2722.

Laviolette SR, Grace AA (2006). Cannabinoids potentiate emotional learning plasticity in neurons of the medial prefrontal cortex through basolateral amygdala inputs. J Neurosci 26: 6458-6468.

Lyons DM, Lopez JM, Yang C, Schatzberg AF (2000). Stress-level cortisol treatment impairs inhibitory control of behavior in monkeys. J Neurosci 20: 7816-7821.

Maroun M, Akirav I (2007). Arousal and stress effects on consolidation and reconsolidation of recognition memory. Neuropsychopharmacology 33: 394-405.

Marsicano G, Wotjak CT, Azad SC, Bisogno T, Rammes G, Cascio MG et al (2002). The endogenous cannabinoid system controls extinction of aversive memories. Nature 418: 530-534.
Moreira FA, Wotjak CT (2010). Cannabinoids and anxiety. Curr Top Behav Neurosci 2: 429-450.

Mulder AB, Hodenpijl MG, da Silva FH (1998). Electrophysiology of the hippocampal and amygdaloid projections to the nucleus accumbens of the rat: convergence, segregation, and interaction of inputs. J Neurosci 18: 5095-5102.

Nestler EJ, Barrot M, DiLeone RJ, Eisch AJ, Gold SJ, Monteggia LM (2002). Neurobiology of depression. Neuron 34: $13-25$.

O'Donnell P, Grace AA (1995). Synaptic interactions among excitatory afferents to nucleus accumbens neurons: hippocampal gating of prefrontal cortical input. J Neurosci 15: 3622-3639.

Patel S, Hillard CJ (2006). Pharmacological evaluation of cannabinoid receptor ligands in a mouse model of anxiety: further evidence for an anxiolytic role for endogenous cannabinoid signaling. J Pharmacol Exp Ther 318: 304-311.

Patel S, Roelke CT, Rademacher DJ, Cullinan WE, Hillard CJ (2004). Endocannabinoid signaling negatively modulates stressinduced activation of the hypothalamic-pituitary-adrenal axis. Endocrinology 145: 5431-5438.

Paxinos G, Watson C (1997). The Rat Brain in Stereotaxic Coordinates: Hard Cover Edition. Academic Press.

Pliakas AM, Carlson RR, Neve RL, Konradi C, Nestler EJ, Carlezon WA (2001). Altered responsiveness to cocaine and increased immobility in the forced swim test associated with elevated cAMP response element-binding protein expression in nucleus accumbens. J Neurosci 21: 7397-7403.

Rademacher DJ, Meier SE, Shi L, Vanessa Ho W-S, Jarrahian A, Hillard CJ (2008). Effects of acute and repeated restraint stress on endocannabinoid content in the amygdala, ventral striatum, and medial prefrontal cortex in mice. Neuropharmacology 54: 108-116.

Ramot A, Akirav I (2012). Cannabinoid receptors activation and glucocorticoid receptors deactivation in the amygdala prevent the stress-induced enhancement of a negative learning experience. Neurobiol Learn Mem 97: 393-401.

Reagan L, Hendry R, Reznikov L, Piroli G, Wood G, Mcewen B et al (2007). Tianeptine increases brain-derived neurotrophic factor expression in the rat amygdala. Eur J Pharmacology 565: 68-75.

Rebec GV, Christensen JR, Guerra C, Bardo MT (1997). Regional and temporal differences in real-time dopamine efflux in the nucleus accumbens during free-choice novelty. Brain Res 776: 61-67.

Roozendaal B, de Quervain DJ-F, Ferry B, Setlow B, McGaugh JL (2001). Basolateral amygdala-nucleus accumbens interactions in mediating glucocorticoid enhancement of memory consolidation. J Neurosci 21: 2518-2525.

Roozendaal B, Hernandez A, Cabrera SM, Hagewoud R, Malvaez M, Stefanko DP et al (2010). Membrane-associated glucocorticoid activity is necessary for modulation of long-term memory via chromatin modification. J Neurosci 30: 5037-5046.

Roozendaal B, McGaugh JL (1996). Amygdaloid nuclei lesions differentially affect glucocorticoid-induced memory enhancement in an inhibitory avoidance task. Neurobiol. Learn Mem 65: 1-8.

Roozendaal B, Okuda S, Zee EAV, der, McGaugh JL (2006). Glucocorticoid enhancement of memory requires arousalinduced noradrenergic activation in the basolateral amygdala. PNAS 103: 6741-6746.

Roozendaal B, Quirarte GL, McGaugh JL (2002). Glucocorticoids interact with the basolateral amygdala beta-adrenoceptor-cAMP/ cAMP/PKA system in influencing memory consolidation. Eur J Neurosci 15: 553-560.

Segev A, Akirav I (2011). Differential effects of cannabinoid receptor agonist on social discrimination and contextual fear in amygdala and hippocampus. Learn Mem 18: 254-259. 
Segev A, Ramot A, Akirav I (2012). Stress hormones receptors in the amygdala mediate the effects of stress on the consolidation, but not the retrieval, of a non aversive spatial task. PLoS One 7: e29988.

Segev A, Rubin AS, Abush H, Richter-Levin G, Akirav I (2014). Cannabinoid receptor activation prevents the effects of chronic mild stress on emotional learning and LTP in a rat model of depression. Neuropsychopharmacology 39: 919-933.

Setlow B, Roozendaal B, McGaugh JL (2000). Involvement of a basolateral amygdala complex-nucleus accumbens pathway in glucocorticoid-induced modulation of memory consolidation. Eur J Neurosci 12: 367-375.

Steiner MA, Wotjak CT (2008). Role of the endocannabinoid system in regulation of the hypothalamic-pituitary-adrenocortical axis. Prog Brain Res 170: 397-432.
Tasker JG, Di S, Malcher-Lopes R (2006). Rapid glucocorticoid signaling via membrane-associated receptors. Endocrinology 147: 5549-5556.

Viveros MP, Marco EM, File SE (2005). Endocannabinoid system and stress and anxiety responses. Pharmacol Biochem Behav 81: 331-342.

Wallace DL, Han M-H, Graham DL, Green TA, Vialou V, Iñiguez SD et al (2009). CREB regulation of nucleus accumbens excitability mediates social isolation-induced behavioral deficits. Nat Neurosci 12: 200-209.

Zaidan H, Leshem M, Gaisler-Salomon I (2013). Prereproductive stress to female rats alters corticotropin releasing factor type 1 expression in ova and behavior and brain corticotropin releasing factor type 1 expression in offspring. Biol Psychiatry 74: 680-687. 